\title{
Therapie der chronischen Hepatitis C bei Patienten mit psychischen Erkrankungen
}

\author{
M. Schäfer
}

Treatment of Chronic Hepatitis C in Mentally III Patients

\section{Zusammenfassung}

70-90\% der Patienten mit einer intravenösen Drogenabhängigkeit sind chronisch mit dem Hepatitis-C-Virus (HCV) infiziert und haben eine Indikation zur antiviralen Therapie mit pegyliertem Interferon- $\alpha$ plus Ribavirin. Eine bei dieser Patientengruppe jedoch häufig vorhandene psychiatrische Komorbidität stellt bei den zahlreichen möglichen psychischen Nebenwirkungen des Interferon- $\alpha$ eine zumindest relative Kontraindikation dar, aufgrund derer die Patienten immer noch häufig von einer Therapie ausgeschlossen werden. Die in diesem Artikel zusammengefasste aktuelle Studienlage zeigt jedoch, dass auch bei Patienten mit einer Drogenabhängigkeit und psychischen Störungen während der HCV-Therapie gleiche Ansprechraten und gute Haltequoten erreicht werden können, wenn die Patienten interdisziplinär betreut werden. Nur schwere und aktuell exazerbierte psychiatrische Krankheitsbilder sollten daher Kontraindikationen gegen eine Therapie mit Interferon- $\alpha$ darstellen.

\section{Schliuisselwörter}

Hepatitis C · Interferon- $\alpha \cdot$ psychische Störungen · Drogenabhängigkeit $\cdot$ Nebenwirkungen

\section{Abstract}

$70-90 \%$ of intravenous drug users are chronically infected with the hepatitis $C$ virus and would need antiviral treatment with pegylated interferon- $\alpha$ and ribavirin. However, because interferon$\alpha$ can induce severe psychiatric side effects, especially the presence of psychiatric comorbidities often tends to result in an exclusion from the otherwise helpful antiviral treatment. Recent trials about hepatitis $C$ treatment for intravenous drug users demonstrate that response rates and adherence do not significantly differ between patients with or without drug addiction, even in case of psychiatric disorders, if patients are treated in an interdisciplinary setting. Thus, only severe and unstable psychiatric conditions should still be regarded as contraindication against antiviral treatment with interferon- $\alpha$.

\section{Key words}

Hepatitis C · Interferon- $\alpha \cdot$ psychiatric disorders · drug addiction . side-effects

\section{Ausgangslage}

Bei einem erheblichen Anteil der mit dem Hepatitis C infizierten Patienten lassen sich auch psychische Erkrankungen finden. Eine Untersuchung an 352 Ambulanzpatienten mit einer chronischen Hepatitis C ergab bei $27 \%$ der Patienten eine zusätzlich psychiatrische Diagnose [1]. 76\% der Patienten hatten eine affektive Störung und $24 \%$ eine Störung aus dem schizophrenen Formenkreis. Letzt- endlich erhielten auch nur 24\% der Patienten tatsächlich eine antivirale Therapie. Auch weitere Arbeiten konnten zeigen, dass bis zu 50\% der Patienten mit psychiatrischen Risikofaktoren unbehandelt blieben, obwohl sie formal die Kriterien für eine Behandlung erfüllten [2 -4]. Umgekehrt konnten für psychisch kranke Patienten deutlich erhöhte HCV-Prävalenzen zwischen 6,8-8,5\% gezeigt werden [5-7]. Das Vorhandensein einer psychischen Erkrankung stellt dabei vor allem für jene Patienten einen Risikofak-

Institutsangaben

Klinik für Psychiatrie und Psychotherapie, Charité - Universitätsmedizin Berlin

Korrespondenzadresse

Martin Schäfer · Klinik für Psychiatrie und Psychotherapie, Charité - Universitätsmedizin Berlin, Campus

Charité Mitte·Schumannstr. 20/21·10117 Berlin·E-mail: martin.schaefer@charite.de

Bibliografie

Suchttherapie 2005; 6: 66 - 69 @ Georg Thieme Verlag KG Stuttgart • New York

DOI 10.1055/s-2005-858335

ISSN 1439-9903 
tor für eine HCV-Infektion dar, die in psychiatrischen Institutionen untergebracht sind oder die z.B. im Rahmen von „Selbstheilungsversuchen“ zeitweise illegale Drogen konsumiert haben.

Gründe für Nichtbehandlung von Patienten mit psychischen Erkrankungen

Unter der das Immunsystem stark stimulierenden Therapie mit IFN- $\alpha$ können verschiedene neuropsychiatrische Nebenwirkungen auftreten. Somit war es naheliegend anzunehmen, dass sich bestehende psychische Vorerkrankungen durch die Behandlung verschlechtern [8]. Zu den wichtigsten Komplikationen gehört das Auftreten depressiver Symptome mit gelegentlich zu beobachtenden Suizidgedanken. In solchen Fällen wurde früher grundsätzlich die Therapie unterbrochen oder zumindest die IFN-Dosis reduziert. Psychisch Kranke wurden im Falle bestehender Depressionen oder bekannter früherer Suizidversuche gar nicht erst anbehandelt. Weiterhin wurde angenommen, dass die Verschlechterung der psychischen Befindlichkeit während der IFN- $\alpha$-Behandlung zu einer verschlechterten Haltquote und einer verminderten Therapiecompliance führt [9-11].

Tatsächlich weiß man aber mittlerweile, dass auch schon die chronische Hepatitis-C-Infektion selbst mit einer Einschränkung der psychischen Lebensqualität aufgrund gehäuft vorkommender Beschwerden wie chronische Müdigkeit und Antriebsverlust, Depressionen und kognitiven Störungen verbunden ist $[8,12]$. Zudem können psychische Nebenwirkungen bei allen Patienten unabhängig von ihrer psychiatrischen Vorgeschichte auftreten [12-14]. Auch die beschriebenen Suizidversuche während einer Interferon-Behandlung waren zwar mit psychischen Veränderungen während der Therapie assoziiert, traten jedoch sowohl bei Patienten mit als auch ohne psychiatrische Risikofaktoren gleichermaßen auf [15]. Patienten ohne frühere psychiatrische Behandlungen sind sogar insofern intensiver zu betreuen, da sie psychische Veränderungen während einer Interferonbehandlung nicht rechtzeitig erkennen und im Vergleich zu „psychiatrieerfahrenen" Patienten seltener und erst spät das Angebot fachkundiger psychiatrischer Hilfe annehmen.

\section{Studienlage}

In einer ersten offenen unkontrollierten Studie beschrieb 1995 Van Thiel mit seiner Arbeitsgruppe, dass in einem interdisziplinären Setting HCV-infizierte Patienten mit Suchterkrankungen und teilweise zusätzlicher psychiatrischer Komorbidität erfolgreich mit Standard IFN- $\alpha$ (5 MU täglich) behandelt werden können [16]. Insgesamt wurden 31 Patienten behandelt, die an affektiven Störungen, schizoaffektiven Störungen oder Erkrankungen aus dem schizophrenen Formenkreis litten. Zusätzlich bestand bei den meisten Patienten eine Suchterkrankung. $48 \%$ der Patienten zeigten eine negative PCR bei Therapieende. Bei nur vier Patienten trat während der Behandlungszeit eine Exazerbation der psychischen Erkrankung auf und nur 2 Patienten brachen die Therapie vorzeitig ab. In einer weiteren Studie verglichen Van Thiel u. Mitarb. 120 HCV-infizierte Patienten mit früherer intravenöser Drogenabhängigkeit mit 120 Kontrollpatienten bezüglich des Ansprechens auf eine IFN- $\alpha$-Monotherapie (5 MU s. c. täglich) [17]. Die Ansprechraten lagen bei 33\% für Patienten mit früherer Drogenabhängigkeit und $37 \%$ bei den Kontrollen. Eine psychiatrische Komorbidität beeinflusste erneut weder die Haltequote noch das Therapieansprechen.

Pariante u. Mitarb. zeigten, dass Hepatitis-C-infizierte Patienten mit affektiven Störungen oder Angsterkrankungen ebenfalls erfolgreich mit Interferon- $\alpha$ behandelt werden können. 25 Patienten mit psychiatrischer Diagnose und 35 psychisch gesunde Kontrollen wurden bezüglich des Therapieansprechens, der Haltequote und der Häufigkeit und Schwere von Depressionen verglichen. Psychiatrische Patienten zeigten dabei keine Unterschiede bezüglich der Ansprechraten, der Haltequote und der Häufigkeit und Schwere von Depressionen oder Angstsymptomen während der antiviralen Therapie $[18,19]$. Während die Gruppe der Patienten mit psychischen Störungen vor der antiviralen Behandlung noch signifikant höhere Ratings bezüglich depressiver Symptome und in der verwendeten Angstskala aufwiesen, verschwanden diese Unterschiede durch einen höheren Anstieg der Skalen in der Kontrollgruppe. Eine weitere kürzlich erschienene Studie konnte ebenfalls zeigen, dass Patienten trotz vorbestehender Depressionen keine oder nur geringe Anstiege der Depressionswerte zeigten. Dagegen waren die Depressionsskalenwerte bei Patienten ohne vorbestehende Depressionen nach 3 und 6 Monaten einer adjuvanten IFN-Behandlung aufgrund eines malignen Melanoms deutlich erhöht [20]. Im Kontrast zu diesen Studien beschrieb Capuron, dass Depressionen bei Behandlungsbeginn als Risikofaktor für die Entwicklung hoher Depressionsscores während der Therapie anzusehen seien [21].

Sylvestre berichtete vorläufige Ergebnisse aus einer Studie an 66 HCV-positiven mit Methadon substituierten Patienten. Die meisten Patienten zeigten eine psychiatrische Komorbidität, wobei Patienten mit akuten Depressionen, Exazerbationen der Grunderkrankungen und Patienten mit aktivem Drogen- oder Alkoholmissbrauch ausgeschlossen wurden. Die Patienten erhielten eine Behandlung mit Standard-Interferon- $\alpha$ (3 MU/Woche) und Ribavirin (1000-1200 mg Tag) [22]. 56\% der Patienten hatten zudem eine psychiatrische Dauermedikation. Am Ende der Behandlung erhielten $88 \%$ eine medikamentöse Behandlung mit Psychopharmaka. 68\% davon nahmen Antidepressiva ein. Insgesamt beendeten 78\% der Patienten die Therapie erfolgreich. 29\% zeigten ein langfristiges virologisches Therapieansprechen.

In einer weiteren prospektiven und kontrollierten Studie wurden Haltequoten, Ansprechraten und insbesondere die Häufigkeit von schweren Depressionen während einer Hepatitis-C-Behandlung HCV-infizierter Patienten mit früherer oder aktueller Suchterkrankung sowie psychisch kranke Patienten mit einer HCV-infizierten Kontrollgruppe, in denen psychische Störungen oder Suchterkrankungen ausgeschlossen wurden, verglichen [14]. Die Behandlung erfolgte mittels Interferon- $\alpha-2$ a und Ribavirin gemäß des Genotypen über 24 bzw. 48 Wochen. Die Patienten wurden interdisziplinär betreut und regelmäßig bezüglich psychiatrischer Nebenwirkungen untersucht. Die Kontrollgruppe bestand aus 23 Patienten, die Gruppen mit Abhängigkeitserkrankungen (methadonsubstituiert bzw. frühere Abhängigkeit) aus jeweils 20 Patienten und die Gruppe mit psychischen Erkrankungen aus 16 Patienten. 6 Patienten litten unter einer affektiven Störung, 3 Patienten wiesen eine Kombination aus einer Borderline-Persönlichkeit und einer affek- 
tiven Störung auf, bei einem Patient wurde eine Angsterkrankung diagnostiziert, 2 Patienten hatten eine schizoaffektive und weitere 4 Patienten eine schizophrene Störung. Alle Patienten waren in nervenärztlicher Behandlung. 38\% hatten depressive Symptome bei Beginn der antiviralen Behandlung, wobei zu diesem Zeitpunkt 19\% eine antidepressive Medikation einnahmen. Während der Therapie entwickelten 6\% der psychiatrischen Patienten und 12\% der Kontrollen neue depressive Episoden. Betrachtet man nur die Häufigkeit der Depressionen unter der antiviralen Therapie mit IFN- $\alpha$, so konnte bei $12 \%$ der Kontrollen und $44 \%$ der psychiatrischen Patienten eine Depression diagnostiziert werden. Kontrollpatienten erhielten in $4 \%$ eine antidepressive Therapie, während $62 \%$ der psychiatrischen Patienten Antidepressiva während der Interferongabe einnahmen. Diese Beobachtung weist auf eine protektive Wirkung der Antidepressiva gegen IFN- $\alpha$-assoziierte Depressionen hin. Psychiatrische Patienten, denen die Antidepressiva vertraut waren, neigten zu einer frühzeitigen Einnahme, während die Kontrollpatienten eher eine medikamentös-antidepressive Behandlung ablehnten. Sechs Monate nach Behandlung wiesen noch 35\% der Kontrollpatienten und 38\% der psychiatrischen Patienten bei ähnlicher Virusgenotypverteilung eine Virusfreiheit bei der PCR-Untersuchung auf (als Kriterium eines langfristigen Therapieansprechens). Die Zahl der Patienten, die vorzeitig die Therapie beenden mussten, war ebenfalls nicht signifikant unterschiedlich in den Gruppen (13\% der Kontrollen und $18 \%$ der Patienten mit psychischen Störungen). Kein Patient mit einer psychischen Erkrankung beendete die Therapie vorzeitig aufgrund von psychiatrischen Nebenwirkungen oder Exazerbationen der Grunderkrankung. Es kam jedoch zu einzelnen stationären Aufenthalten zur Therapieoptimierung oder bei Verschlechterungen im Rahmen der psychischen Erkrankung, die jedoch nicht auf die Interferongabe ursächlich zurückzuführen war.

Eine prospektiv-klinische Studie untersuchte sogar, ob sich Interferon- $\alpha$ direkt zur Therapie von schizophrenen Störungen eignet. Tatsächlich benötigten die zusätzlich mit IFN- $\alpha$ behandelten Patienten weniger Neuroleptika als die Kontrollen ohne additives IFN- $\alpha$ [23]. Weitere Kasuistiken zeigen, dass schizophrene Patienten mit einer chronischen HCV-Infektion erfolgreich mit IFN- $\alpha$ behandelt werden können, jedoch eine interdisziplinäre Betreuung und je nach Art der psychiatrischen Basismedikation aufgrund möglicher hämatologischer Interaktionen ein engmaschiges Monitoring benötigen [24-26].

Zusammenfassend weisen die Ergebnisse der in den letzten Jahren durchgeführten Studien darauf hin, dass eine psychische Erkrankung per se kein Risikofaktor für ein gehäuftes Auftreten neuropsychiatrischer Nebenwirkungen von Interferon- $\alpha$ ist. Auch zeigten sich keine Unterschiede bezüglich der Ansprechraten und Haltequoten von Patienten mit psychischen Erkrankungen und psychisch gesunden Kontrollen. Allerdings sollte eine interdisziplinäre Betreuung des Patienten immer gewährleistet sein. Die bei diesen Patienten oft vorbestehende oder rechtzeitig angesetzte antidepressive Medikation reduzierte sogar in vielen Fällen die Häufigkeit von depressiven Veränderungen während der IFN$\alpha$-Therapie gegenüber psychisch gesunden Kontrollen, die diese Medikation nicht hatten. Dass eine antidepressive Vorbehandlung auch prophylaktisch gegen IFN-assoziierte Depressionen schützen kann, wurde kürzlich bei Patienten mit malignem Melanom gezeigt [27]. Trotzdem werden weitere prospektive kontrollierte Stu- dien an größeren Kollektiven benötigt, um die bisherigen Daten zu belegen.

Wer sollte aus psychiatrischen Grüinden eher nicht behandelt werden?

Wenn eine interdisziplinäre Zusammenarbeit zwischen HCV-Behandlern und Psychiatern bzw. Suchtmedizinern sichergestellt ist, verbleiben letztendlich nur noch wenige Gründe, die gegen eine Behandlung der Hepatitis C mit IFN- $\alpha$ sprechen könnten [28]. Schwere akute bestehende Exazerbationen psychischer Erkrankungen (darunter depressive Episoden, manische Episoden, akute paranoide Psychosen, exazerbierte Persönlichkeitsstörungen oder Panikstörungen, schwere Impulskontrollstörungen etc.) sollten dazu führen, dass die Patienten zunächst durch eine adäquate psychiatrische Therapie stabilisiert werden. Erst dann sollte bei bei entsprechendem Wunsch des Patienten in Absprache mit den behandelnden Ärzten eine antivirale Therapie der chronischen Hepatitis C erwogen werden. Auch die psychosoziale Situation ist stets in die Entscheidung mit einzubeziehen (fester Wohnsitz, soziale Kontakte). Für isolierte Patienten ohne eine Bezugsperson sollte zunächst ein adäquates soziales Umfeld geschaffen werden. Auch psychiatrische Hilfesysteme (sozialpsychiatrischer Dienst, betreutes Wohnen, Pflegedienste, Tagesstätten usw.) können und sollten vermehrt in die Behandlung mit einbezogen werden. Eine Vorgeschichte von eventuell sogar mehreren Suizidversuchen oder entsprechender familiärer Vorbelastung bedarf in jedem Fall einer intensiven psychiatrischen Abklärung. Die Entscheidung für eine Therapie sollte vor allem bei Patienten mit schweren Persönlichkeitsstörungen und Neigung zu impulsiven, unkontrollierten Handlungen mit entsprechender Vorsicht getroffen werden.

Nicht behandelt werden sollten Patienten mit schweren hirnorganischen Veränderungen (Demenzen, organischen Wesensänderungen etc.) [29]. Auch fehlende Motivation und ein unkontrollierter Alkoholmissbrauch sprechen gegen eine Therapie. Patienten mit Alkoholmissbrauch während der Therapie sprechen deutlich schlechter auf die Behandlung mit Interferonen an, zeigen aber auch eine schlechtere Leberhistologie mit schneller Progression zur Leberzirrhose und ein erhöhtes Risiko für die Entwicklung eines Leberzellkarzinoms. Während die Interaktionen von Alkohol mit den Hepatitis-C-Viren nicht vollends aufgeklärt sind, zeigen einige Studien eine Veränderung der Quasipezies, eine Beeinflussung der Virusreplikation und eine Schwächung des gesamten Immunsystems [30-35]. Umso wichtiger erscheint eine rasche Zuführung solcher Patienten in eine adäquate suchtmedizinische Behandlung der Alkoholabhängigkeit, um die rasche Progression der Lebererkrankung aufzuhalten und bei entsprechender Stabilisierung doch noch eine antivirale Therapie einleiten zu können.

\section{Literatur}

${ }^{1}$ Lynch P, Peters M, Quigley M et al. Impact of psychiatric disorders on treatment of patients with hepatitis C. Gastroenterol 1998; 114 (Suppl.): A1294

2 Shad J, Person J, Brann O. How often are referred chronic hepatitis C patients candidates for antiviral therapy. Hepatology 2000; 32: A283 
${ }^{3}$ Taruschio G, Santarini G, Sica G. Psychiatric disorders in hepatitis C virus related chronic liver disease. Gastroenterology 1996; 110: A1342

${ }^{4}$ Taylor LE, Costello T, Alt E et al. Psychiatric illness and illicit drugs as barriers to hepatitis $C$ treatment among HIV/hepatitis C virus co-infected individuals. AIDS 2002; 16 (12): 1700-1701

${ }^{5}$ Dinwiddie SH, Shicker L, Newman T. Prevalence of hepatitis C among psychiatric patients in the public sector. Am J Psychiatry 2003; 160 (1): $172-174$

${ }^{6}$ Cividini A, Pistorio A, Regazzetti A et al. Hepatitis C virus infection among institutionalised psychiatric patients: a regression analysis of indicators of risk. J Hepatol 1997; 27 (3): 455-463

${ }^{7}$ Chang TT, Lin H, Yen YS et al. Hepatitis B and hepatitis C among institutionalized psychiatric patients in Taiwan. J Med Virol 1993; 40 (2): $170-173$

${ }^{8}$ Schaefer M, Engelbrecht MA, Gut O et al. Interferon alpha (IFNalpha) and psychiatric syndromes: a review. Prog Neuropsychopharmacol Biol Psychiatry 2002; 26 (4): 731 - 746

${ }^{9}$ McDonald EM, Mann AH, Thomas HC. Interferons as mediators of psychiatric morbidity. An investigation in a trial of recombinant alpha-interferon in hepatitis-B carriers. Lancet 1987; 2 (8569): 1175-1178

${ }^{10}$ Renault PF, Hoofnagle JH, Park Y et al. Psychiatric complications of long-term interferon alfa therapy. Arch Intern Med 1987; 147 (9): $1577-1580$

${ }^{11}$ Renault PF, Hoofnagle JH. Side effects of alpha interferon. Semin Liver Dis 1989; 9 (4): 273-277

12 Valentine AD, Meyers CA, Kling MA et al. Mood and cognitive side effects of interferon-alpha therapy. Semin Oncol 1998; 25 (1 Suppl 1): $39-47$

${ }^{13}$ Dieperink E, Willenbring M, Ho SB. Neuropsychiatric symptoms associated with hepatitis $C$ and interferon alpha: A review. Am J Psychiatry 2000; 157 (6): 867-876

${ }^{14}$ Schaefer M, Schmidt F, Folwaczny C et al. Adherence and mental side effects during hepatitis $C$ treatment with interferon alfa and ribavirin in psychiatric risk groups. Hepatology 2003; 37 (2): $443-451$

15 Janssen HL, Brouwer JT, van der Mast RC et al. Suicide associated with alfa-interferon therapy for chronic viral hepatitis. J Hepatol 1994; 21 (2): $241-243$

${ }^{16}$ Van Thiel DH, Friedlander L, Molloy PJ et al. Interferon-alpha can be used successfully in patients with hepatitis $C$ virus-positive chronic hepatitis who have a psychiatric illness. Eur J Gastroenterol Hepatol 1995; 7 (2): $165-168$

${ }^{17}$ Van Thiel DH, Anantharaju A, Creech S. Response to treatment of hepatitis $C$ in individuals with a recent history of intravenous drug abuse. Am J Gastroenterol 2003; 98 (10): 2281-2288

${ }^{18}$ Pariante CM, Orru MG, Baita A et al. Treatment with interferon-alpha in patients with chronic hepatitis and mood or anxiety disorders. Lancet 1999; 354 (9173): $131-132$
${ }^{19}$ Pariante CM, Landau S, Carpiniello B. Interferon alfa-induced adverse effects in patients with a psychiatric diagnosis. N Engl J Med 2002; 347 (2): $148-149$

${ }^{20}$ Schaefer M, Schmidt F, Horn M et al. Depression during treatment with interferon alpha. Psychosomatics 2004; 45 (2): 176

${ }^{21}$ Capuron L, Ravaud A. Prediction of the depressive effects of interferon alfa therapy by the patient's initial affective state. N Engl J Med 1999; 340 (17): 1370

22 Sylvestre DL. Treating hepatitis $C$ in methadone maintenance patients: an interim analysis. Drug Alcohol Depend 2002; 67 (2): $117-123$

${ }^{23}$ Cabrera Gomez JA Jr, Cordero G, Fernandez LO et al. Treatment of schizophrenic disorder, paranoid type, with intramuscular recombinant alpha-2 b interferon. Biotherapy 1993; 7 (1): 27-37

${ }^{24}$ Dobmeier M, Frick E, Frank S et al. Schizophrenic psychosis: a contraindication for treatment of hepatitis $C$ with interferon alpha? Pharmacopsychiatry 2000; 33 (2): $72-74$

${ }^{25}$ Schafer M, Boetsch T, Laakmann G. Psychosis in a methadone-substituted patient during interferon-alpha treatment of hepatitis $C$. Addiction 2000; 95 (7): $1101-1104$

${ }^{26}$ Schafer M, Schmidt F, Grunze H et al. [Interferon alpha-associated agranulocytosis during clozapine treatment. Case report and status of current knowledge]. Nervenarzt 2001; 72 (11): $872-875$

${ }^{27}$ Musselman DL, Lawson DH, Gumnick JF et al. Paroxetine for the prevention of depression induced by high-dose interferon alfa. N Engl J Med 2001; 344 (13): 961 - 966

${ }^{28}$ Schäfer M. Hepatitis-C-Therapie: Management psychiatrischer Nebenwirkungen von Interferon alfa. Stuttgart, New York: Georg Thieme Verlag, 2004

${ }^{29}$ Schafer M, Schwaiger M. [Incidence, pathoetiology and treatment of interferon-alpha induced neuro-psychiatric side effects]. Fortschr Neurol Psychiatr 2003; 71 (9): 469-476

${ }^{30}$ Anand BS, Velez M. Influence of chronic alcohol abuse on hepatitis C virus replication. Dig Dis 2000; 18 (3): 168 - 171

${ }^{31}$ Anderson S, Nevins CL, Green LK et al. Assessment of liver histology in chronic alcoholics with and without hepatitis $C$ virus infection. Dig Dis Sci 2001; 46 (7): $1393-1398$

32 Bhattacharya R, Shuhart MC. Hepatitis C and alcohol: interactions, outcomes, and implications. J Clin Gastroenterol 2003; 36 (3): $242-252$

${ }^{33}$ Jamal MM, Morgan TR. Liver disease in alcohol and hepatitis C. Best Pract Res Clin Gastroenterol 2003; 17 (4): 649-662

${ }^{34}$ Peters MG, Terrault NA. Alcohol use and hepatitis C. Hepatology 2002; 36 (5 Suppl 1): S220-S225

${ }^{35}$ Zhang T, Li Y, Lai JP et al. Alcohol potentiates hepatitis C virus replicon expression. Hepatology 2003; 38 (1): $57-65$ 